\title{
Apresentação - Pesquisas sobre manuais escolares e mídias educativas: tensões e interações entre impresso e digital
}

\author{
Tânia Maria F. Braga Garcia* \\ Jesús Rodríguez Rodríguez ${ }^{* *}$ \\ Alessandra Anichini***
}

O propósito fundamental deste dossiê é apresentar tendências e abordagens atuais na produção de conhecimento sobre manuais escolares e mídias educativas no Brasil e em outros países, evidenciando resultados de pesquisas, mas também identificando lacunas ou temas menos privilegiados que poderão orientar novas investigações. Igualmente, pretende-se contribuir com o debate teórico sobre a produção, circulação e apropriação de manuais e mídias educativas, sejam impressas ou digitais, em diferentes contextos da contemporaneidade.

Os manuais escolares são, reconhecidamente, materiais que afetam a vida cotidiana das escolas, de diferentes formas e em diferentes situações. No Brasil em especial, a existência de programas nacionais de avaliação e distribuição gratuita de livros didáticos impressos a todos os alunos de ensino fundamental e médio, na maior parte das disciplinas curriculares, coloca o debate sobre o tema como uma questão política e educativa da maior relevância. Essa particularidade justifica a necessidade e a pertinência de pesquisas no campo educacional.

Em outros países, que possuem outras formas de produzir e colocar em circulação os manuais escolares, diferentes dimensões ganham relevância, como, por exemplo, a dimensão econômica do tema. Embates entre os resultados dos processos avaliativos realizados por pesquisadores e a qualidade dos manuais produzidos pelas editoras comerciais são notadamente uma questão que ocupa a atenção da academia, das escolas e da mídia em lugares como a Espanha, por exemplo.

* Universidade Federal do Paraná. Programa de Pós-Graduação em Educação. Curitiba, Paraná, Brasil. E-mail: taniabraga@pq.cnpq.br. https://orcid.org/0000-0003-0614-290X.

${ }^{* *}$ Universidad de Santiago de Compostela. Santiago de Compostela. España. E-mail: jesus. rodriguez.rodriguez@usc.es. https://orcid.org/0000-0003-4194-2574.

${ }^{* * * *}$ Istituto Nazionale Documentazione, Innovazione e Ricerca Educativa (INDIRE). Firenze, Italia. E-mail: a.anichini@indire.it. https://orcid.org/0000-0002-3319-1689. 
Nesse mesmo país, os últimos anos mostraram uma valorização de questões relacionadas à produção e à circulação de recursos digitais, que se expressam em publicações temáticas tais como a Relatec - Revista Latinoamericana de Tecnologia Educativa (ver o números temático 16-2, 2017), a Revista Profesorado (monográfico 20-1, 2016) e o número 20 da @tic revista d'educació educativa, que evidenciam a emergência deste âmbito de estudos com diversidade de enfoques e perspectivas. Pesquisas nacionais têm sido financiadas pelo governo espanhol para compreender as questões relativas às tecnologias de informação e comunicação e sua presença na vida escolar, assim como redes de investigadores no país e na comunidade europeia têm se dedicado ao estudo do tema, interessando-se em conhecer o ponto de vista de professores, alunos e pais sobre os materiais disponibilizados.

Também na Itália as relações entre as mídias impressas e as digitais estão em foco nas atividades do Istituto Nazionale di Documentazione, Innovazione e Ricerca Educativa (INDIRE), centro ligado ao Ministero dell' Istruzione, que desenvolve atividades de pesquisa e de formação de professores. Além disso, o centro participa dos processos de avaliação do sistema educativo e dispõe de um precioso arquivo histórico pedagógico. Em particular, o desenvolvimento de atividades de produção de materiais pelas escolas é uma linha de pesquisa e formação que abre perspectivas de diálogo entre os diferentes tipos de suportes que podem ser utilizados nos recursos para ensinar e aprender no mundo contemporâneo.

As investigações do INDIRE, que tem sua sede principal em Firenze, objetivam a melhoria da didática no sistema educativo italiano, por isso, se espraiam para aspectos diversos da relação entre digital e impresso e focalizam diferentes níveis de ensino, diferentes tipos de escola por meio de projetos específicos, como o "Avanguardie Educative". Trata-se de um projeto de pesquisa-ação destinado a repensar a organização didática, o tempo e o espaço escolar. As escolas são apoiadas em suas ações de desenvolvimento curricular, respeitando os espaços de autonomia para experimentações pedagógicas e, por consequência, para a produção de recursos.

No complexo universo de possibilidades para organizar os processos de ensinar e aprender, nos diferentes contextos da contemporaneidade, os suportes digitais têm ocupado a atenção das comunidades escolares e da comunidade acadêmica. No entanto, a presença das tecnologias não retirou da vida de alunos, professores e famílias a presença dos manuais escolares em suporte impresso, que continuam a ser produzidos e a circular, ainda que com diferentes intensidades.

Como objeto de investigação, os manuais escolares - assim como outros materiais didáticos - ganharam importância nos últimos 30 anos, no Brasil e em outros países, como apontam Børre Johnsen (1996), Batista (2002), Choppin 
(2004), Guereña, Ossenbach e Pozzo (2006), Munakata (2012), Escolano Benito (2006), Garcia (2014), Rodríguez, Bruillard e Horsley (2015), Fuchs e Bock (2016), Garcia, N. (2017), Rodríguez Rodríguez, Garcia e Bruillard (2019). Inicialmente valorizados como fontes no âmbito dos estudos históricos, sua forte presença na cultura escolar também demandou estudos de natureza didática e epistemológica que, lentamente, vêm ganhando espaço entre os investigadores e grupos de pesquisa.

Tais pesquisas buscam maior aproximação com o espaço escolar, para compreender as relações que professores e alunos estabelecem com os manuais e outros materiais didáticos, ouvindo esses sujeitos e seus pontos de vista; contudo, elas também se voltam à análise da produção, das formas de avaliação e seleção desses recursos, e das apropriações que os sujeitos escolares fazem ao incluí-los nas atividades de ensino e aprendizagem.

Se no âmbito dos estudos didáticos gerais estas perspectivas têm relevância e são valorizadas pela pesquisa científica, também se deve destacar que o tema se articula a investigações que ocorrem no âmbito das didáticas específicas, abrindo espaços para pesquisas sobre os manuais e materiais didáticos no que se refere às questões epistemológicas dos diferentes campos de conhecimento que produziram e produzem as disciplinas escolares.

Aceita-se que os livros e outros materiais constituem-se como objetos $d a$ cultura escolar, conceito apropriado a partir de Forquin (1993, p. 167) no sentido de que devem ser entendidos na relação com o "conjunto dos conteúdos cognitivos e simbólicos que, selecionados, organizados, 'normalizados', 'rotinizados', sob o efeito dos imperativos da didatização, constituem habitualmente o objeto de uma transmissão deliberada no contexto das escolas". Assim, o conteúdo veiculado por esses objetos expressa os resultados de um processo de seleção cultural a partir do qual são definidos tanto os significados da escolarização como o que se considera relevante ensinar.

Portanto, os livros escolares e outros recursos didáticos materializam o que uma sociedade - ou parte dela - considera valioso transmitir às gerações mais novas no que se refere especialmente aos conhecimentos científicos, estes também entendidos como produto de uma construção histórica e social. Entretanto, como destacado por Dominique Julia (2001), a escolarização não se subordina apenas ao conjunto de normas que definem conhecimentos a ensinar; a cultura escolar inclui um conjunto de práticas que permitem a transmissão desses conhecimentos e a incorporação desses comportamentos.

Com tal entendimento, o conceito de cultura escolar permite considerar os livros e outras mídias educativas que apoiam os processos de escolarização como artefatos dessa cultura; assim, interessa à pesquisa educacional explicitar a compreensão não apenas sobre os conhecimentos científicos que eles intentam 
transmitir, mas também sobre as formas que são por eles privilegiadas em cada tempo e lugar, para ensinar e aprender. Importa também conhecer os valores, entendidos enquanto escolhas sociais e culturais que, explicitadas ou não, sustentam as propostas veiculadas pelos manuais e outros materiais didáticos e cujo objetivo é, ao final, transmitir às novas gerações parte do material simbólico significativo para aquele grupo social.

Mas, talvez a dimensão de análise de maior complexidade - dos livros ou de outros materiais - seja a que está relacionada à sua existência na vida das escolas. Nesse âmbito, materiais são apropriados pela escola, pelos sujeitos, e passam a compor o conjunto de elementos que constituem a vida escolar, em suas múltiplas dimensões. Nesse espaço são estabelecidas definições que dependem das ações dos sujeitos escolares, especialmente de professores e alunos, perspectiva pouco explorada pela pesquisa segundo autores como Choppin (2004), Fernandez Reiris (2005), Martinez, Valls e Piñeda (2009), Garcia (2013), Chaves (2015).

Por outro lado, o desenvolvimento das tecnologias digitais e sua chegada ao mundo escolar nas últimas décadas, mesmo nos países menos desenvolvidos, provocam novas questões sobre os recursos didáticos a serem estudadas pela pesquisa educacional. Registra-se nos últimos anos uma retomada de interesse dos pesquisadores sobre o tema para responder a tais questões, seja do ponto de vista do uso de materiais digitais pelos professores e alunos, seja também sobre os efeitos das novas formas de comunicação e de informação sobre os processos de ensino e aprendizagem, escolar ou em outros âmbitos.

Ainda por efeito dessas tecnologias, abrem-se espaços para a produção de livros e outros materiais em condições nunca antes experimentadas. Os sujeitos escolares podem, nessas novas possibilidades, assumirem-se efetivamente como autores de conteúdos digitais e também de obras que podem ser disponibilizadas virtualmente e de acesso gratuito aos interessados. O que as pesquisas vêm analisando quanto a plataformas digitais e outros recursos, para além dos impressos? O quanto isso tem afetado os processos de produção e circulação de recursos didáticos? E quanto aos processos de ensinar e aprender nessas outras condições - há mudanças em curso?

Esse conjunto de considerações e questões, embora sem esgotar as possibilidades que se abrem à pesquisa, dada a complexidade do tema, pode ser suficiente para justificar a proposição de um dossiê temático. Reconhecendo-se os limites impostos pelas normas da publicação quanto ao número de artigos possíveis, buscou-se dar visibilidade aos temas e perspectivas de investigação que estão em pauta em alguns países nos quais os autores e as autoras têm desenvolvido e compartilhado suas atividades de produção de conhecimento. 
Finalmente, destaca-se a diversidade de investigadores de grupos ou institutos de pesquisa que colaboram na produção deste volume. Parte dos pesquisadores do grupo espanhol está articulada em torno de projeto aprovado e financiado pelo Governo daquele país; o grupo italiano pertence ao reconhecido centro de investigação INDIRE, ligado ao Ministério da Educação, que se ocupa de investigações sobre manuais e mídias digitais e suas relações com a formação de professores; e os autores brasileiros são participantes de centros de ensino e pesquisa em duas diferentes regiões do Brasil (Sul e Sudeste), desenvolvendo projetos conjuntos no Núcleo de Pesquisa em Publicações Didáticas da Universidade Federal do Paraná, em diferentes temáticas que constituem o escopo do trabalho nesse núcleo.

Dada a abrangência e complexidade do tema e os limites de contribuições possíveis, a preocupação dos organizadores foi estruturar eixos relevantes na pesquisa neste campo temático que, assim, de diferentes formas, transversalizam os debates nos diferentes artigos que compõem o dossiê: as relações impressodigital; os desafios da avaliação e da produção de livros e outros materiais, inclusive pelas escolas; as relações dos materiais e mídias educativas com o processo de ensino e aprendizagem e com a formação de professores. Os eixos foram escolhidos em função das problemáticas que se colocam, contemporaneamente, para o processo de escolarização das novas gerações e que desafiam a pesquisa educacional no que tange à presença dos manuais e mídias educativas na vida escola.

Três artigos estão articulados em torno de um mesmo projeto de investigação intitulado "La escuela de la sociedad digital: análisis y propuestas para la producción y uso de los contenidos digitales educativos". Financiado pelo Programa Estatal de I+D+i, o projeto é dirigido pelo Professor Manuel Area Moreira. Pode-se entender que estes três artigos são transversalizados especialmente pelo eixo das relações entre impresso e digital e, em segundo plano, pelo eixo da avaliação e da produção de materiais, neste caso com uma focalização específica nos usuários privilegiados, ou seja, professores e alunos. Transversalizado também pelo eixo da avaliação e produção de materiais, um quarto artigo dedica atenção ao que se informa sobre eles em blogs e páginas da web de Centros de Educação Infantil na Coruña.

O grupo de pesquisadores italianos está articulado às atividades do INDIRE, o Instituto Nacional de Documentação, Inovação e Pesquisa Educacional ligado ao Ministério da Educação, criado em 1925 e ponto de referência para a pesquisa educacional na Itália, e da Università di Firenze. Os artigos do grupo estão transversalizados pelo eixo das relações entre impresso e digital e pelas relações com a formação de professores, foco privilegiado pelas ações do INDIRE a partir das pesquisas sobre inovação educativa. Por sua vez, a Univesità 
di Firenze tem trabalhado as relações entre impresso e digital nas práticas de estudo dos alunos universitários.

Os dois artigos brasileiros estão articulados em torno de atividades realizadas pelo Núcleo de Pesquisas em Publicações Didáticas (NPPD) da Universidade Federal do Paraná. Os estudos do grupo têm sido desenvolvidos no âmbito do diálogo entre a Didática Geral e as Didáticas Específicas e com focalizações diversas, seja no exame de questões relacionadas aos próprios livros e materiais didáticos, seja na análise de relações entre tais materiais e outros elementos do processo de escolarização. Neste caso, os dois textos são transversalizados pelos processos de ensino e aprendizagem e de formação de professores.

Com efeitos de síntese e apresentação, indicam-se a seguir brevemente o conteúdo e o significado dos artigos recebidos.

$\mathrm{O}$ primeiro artigo tem o título Análisis de materiales educativos digitales ofertados desde un portal de contenidos abiertos. El caso de Canarias, de autoria de Carlos González Ruiz (Universidad de La Laguna/ES) e Elena Chirino Alemán (Universidad de Las Palmas de Gran Canaria/ES). Os autores apresentam os resultados da análise de um portal web denominado Eco Escuela 2.0 mantido pela administracão educativa (Consejería de Educación del Gobierno de Canarias-España), caracterizado polo acesso e uso livre do mesmo pelo professorado. Além disso, também são analisados os resultados da análise de uma amostra dos conteúdos ou de materiais didáticos digitais existentes no portal para a Educação Primária.

O segundo texto é intitulado Visiones de los agentes envolvidos en la concepción, divulgación e utilización de materiales educativos digitales en portales educativos españoles, de autoria de José Peirats (Universidad de Valencia/ES), Raúl Eirín (Universidad de Santiago de Compostela/ES) e Jesús Rodríguez Rodríguez (Universidad de Santiago de Compostela/ES). Este artigo apresenta os resultados de pesquisa que recolheu as opiniões do professorado, dos alunos e das famílias sobre materiais digitais que estão disponibilizados em portais institucionais de duas comunidades autônomas da Espanha, a Galícia e Valência, apontando sugestões para seu aperfeiçoamento e melhoria.

Também na direção de compreender o ponto de vista dos alunos, segue-se o texto New study practices. Survey on the use of new and old technologies among university students, com autoria de Ilaria Marchionne (Center for Generative Communication-Department of Political and Social Sciences, University of Florence), Rudi Bartolini (INDIRE) e Taisa Rodrigues Dantas (Universidad de Salamanca, ES). A pesquisa investiga e descreve as práticas de estudo de universitários, fazendo referência ao uso integrado de antigos e novos modos de comunicação, de textos tradicionais e de plataformas e ferramentas digitais comumente utilizadas. Focaliza em particular a relação que, por meio das novas 
ferramentas, se estabelece entre o aluno e o professor, entre o aluno e os colegas e entre o aluno e os textos de estudo, discutindo como o uso de novos métodos de comunicação ou novas formas de textualidade pode favorecer ou inibir a comunicação em um ambiente universitário.

O quarto artigo apresentado intitula-se Las visiones del alumnado sobre los Materiales Didácticos Digitales en España, de autoria de Laura Rego-Agraso (Universidad de A Coruña/ES) e Diana Marín Suelves (Universidad de Valencia/ ES). O texto apresenta os resultados fundamentais da investigação centrada em conhecer a percepção dos alunos dos últimos cursos da Educação Primária sobre a introdução de materiais didáticos digitais (MDD) nas aulas. No estudo, foram realizadas entrevistas grupais com alunos de três comunidades autônomas do estado espanhol. Os resultados permitem identificar os pontos fortes, as dificuldades e as propostas de melhoria dos materiais desde a perspectiva dos principais usuários desses Materiais Didáticos Digitais. O artigo explora os resultados dos estudos realizados em Galícia e Valência.

Ampliando o olhar para as escolas de Educação Infantil, na perspectiva de uma educação inclusiva, o quinto texto apresenta os resultados de investigação que teve por objetivo identificar os tipos de materiais projetados nos blogs e sites dos centros educacionais de educação infantil na Galícia, Espanha. Intitulado Los materiales usados en escuelas de educación infantil proyectados en sus webs y blogs, o artigo tem autoria de Maria Montserrat Castro Rodríguez, da Universidade da Coruña, Espanha. Entre outros resultados, a investigação contribui para evidenciar formas pelas quais os centros educativos participantes utilizam seus blogs e websites para informar e compartilhar as finalidades do uso desses instrumentos, materiais que são divulgados e diferenças entre os centros rurais e urbanos.

As escolas e seu papel na produção do conhecimento são questões centrais no artigo de autoria de Alessandra Anichini e Laura Parigi intitulado Re-writing knowledge, re-design textbooks: auto-production of contents in the classroom. As autoras relatam pesquisa qualitativa realizada no âmbito do Projeto "Avanguardie Educative", que resulta de uma iniciativa conjunta entre o INDIRE e escolas que, de forma autônoma, organizam e propõem formas de transformação do ensino com apoio das tecnologias educativas. $\mathrm{O}$ artigo descreve e analisa a experiência de um grupo de professores italianos em relação à prática de autoprodução de livros didáticos e recursos digitais. A pesquisa, realizada com algumas escolas envolvidas em um processo de experimentação de alternativas à adoção do livro didático, discute as motivações que levam os professores a optarem por essa escolha, oferecendo uma releitura das relações entre currículo, Orientações Nacionais e autonomia de ensino. 
A relação entre os manuais escolares e os processos de formação docente que transversaliza todo o conjunto de textos deste dossiê - assume centralidade no sétimo texto, intitulado Manuais para professores: conceituações e perspectivas de investigação, de autoria de Tânia Maria F. Braga Garcia, do Programa de Pós-Graduação em Educação da Universidade Federal do Paraná, e Vivian Batista da Silva, da Faculdade de Educação da Universidade de São Paulo. O artigo tem o objetivo de contribuir com o campo de produção de conhecimento sobre manuais destinados à formação de professores, em uma perspectiva ainda pouco explorada na pesquisa educacional. Denominados usualmente de manuais pedagógicos, eles têm sido publicados no Brasil desde 1870 com a intenção de oferecer aos futuros professores uma formação pedagógica. A complexificação da Didática como campo de conhecimento e o fortalecimento das Didáticas Específicas produziram transformações nesse conjunto de obras, demandando revisões conceituais e novas perspectivas de investigação, que foram mapeadas pelas autoras a partir de suas pesquisas realizadas nos últimos quinze anos.

Finalizando o conjunto de artigos, Edilson Aparecido Chaves, do Instituto Federal do Paraná - Campus Curitiba, destaca contribuições que a etnografia pode dar às pesquisas sobre a presença dos livros na vida escolar e, em especial, na produção das aulas. Intitulado O livro didático e sua presença em aulas de História: contribuições da etnografia, o autor apresenta pesquisa que enfrentou o desafio de realizar a aproximação com o espaço das aulas, propondo-se a investigar o ponto de vista dos alunos do Ensino Médio sobre os livros didáticos de História incluídos no Programa Nacional do Livro Didático (PNLD) e relações que são estabelecidas com o conhecimento histórico a partir do livro didático. Com base nos resultados, o autor destaca as contribuições que essa forma de investigação pode trazer não só para a compreensão das dinâmicas de uso dos livros didáticos e outros materiais, mas também para seus processos de avaliação, nas dimensões didática e epistemológica.

Ao finalizar a apresentação do dossiê, reassalta-se que os manuais escolares continuam sendo recursos com intensa presença nas práticas educativas das escolas e que, de diferentes formas, influenciam as decisões de caráter pedagógico e didático. Igualmente, pretende-se ressaltar que as linhas de investigação sobre os livros e outros materiais didáticos também têm apresentado diferentes enfoques e perspectivas e, nessa direção, a proposta dos organizadores foi colocar em relevo o tema dos materiais digitais, que ocupa atualmente boa parte dos debates nesse campo temático.

A realidade da convivência no âmbito escolar entre os suportes impressos e digitais, a diversidade de usos que os sujeitos escolares fazem dos materiais disponíveis, as condições de produção e circulação de manuais, o acesso a materiais e conteúdos digitais, as formas de apropriação pelas escolas - estas 
são apenas algumas das questões que demandam atenção de pesquisadores e de formadores de professores, em diferentes países.

A intenção do dossiê é, portanto, apresentar resultados das pesquisas realizadas pelos autores e autoras em torno de algumas dessas questões, entendendo que elas não apenas lançam luzes em determinados pontos dessa complexa rede de relações nas quais os materiais didáticos impressos e digitais estão situados, mas também porque abrem espaços para novas perguntas, novas provocações e novos desafios aos pesquisadores interessados no tema.

Desejamos que o diálogo com os leitores possa ser alimentado pelas contribuições aqui apresentadas.

Em Curitiba, na primavera de 2019.

\section{REFERENCIAS}

Batista, Antônio Augusto Gomes. (2002). Um objeto variável e instável: texto, impressos e livros didáticos. In: Abreu, Márcia Azevedo de (Org.). Leitura, história e história da leitura. Campinas: Mercado de Letras, p. 529-575.

Børre Johnsen, Egil. (1996). Libros de texto en el calidoscópio. Barcelona: PomaresCorredor.

Chaves, Edilson Aparecido. (2015). A presença do livro didático de história em aulas do ensino médio: estudo etnográfico em uma escola do campo. Curitiba: PPGE-UFPR.

Choppin, Alain. (2004). História dos livros e das edições didáticas: sobre o estado da arte. Educação e Pesquisa, 30(3), 549-566.

Escolano Benito, Augustín. (2006). Currículum editado y sociedad del conocimiento: texto, multimedialidad y cultura de la escuela. Valencia: Tirant lo Blanch.

Fernandez Reiris, A. (2005). La importancia de ser llamado libro de texto: hegemonía y control del curriculum en el aula. UNP: Mino y Dávila Editores.

Forquin, Jean-Claude. (1993). Escola e cultura: as bases sociais e epistemológicas do conhecimento escolar. Trad. Guacira Lopes Louro. Porto Alegre: Artes Médicas.

Fuchs, E.; Bock, A. (Ed.). (2016). The Palgrave Handbook of Textbook Studies. New York: Palgrave MacMillan.

Garcia, Nilson M. Dias. (Org.). (2017). O livro didático de física e de ciências em foco: dez anos de pesquisa. São Paulo: Editora Livraria da Física, 91-102. 
Garcia, Tânia M. F. Braga. (2013). Os livros didáticos na sala de aula. In: Garcia, Tânia Maria F. Braga; Schmidt, Maria Auxiliadora; Valls, Rafael. (Orgs.). Didática, História e manuais escolares: contextos ibero-americanos. 1. ed. Ijuí, RS: Editora Unijuí, 2013, v. 6, p. 69-102.

Garcia, Tânia M. F. Braga. (2014). Criteria used by teachers in Brazilian elementary schools in the process of textbook selection. Orbis Scholae (on line), 8(2), 9-22.

Guereña, J. L.; Ossenbach, G.; Pozzo, M. (Dir.). (2006). Manuales escolares en España, Portugal y América Latina (siglos XIX y XX). Madrid: Universidad Nacional de Educación a Distancia.

Julia, Dominique. (2001). A cultura escolar como objeto histórico. Revista Brasileira de História da Educação, 1(jan./jun.). p. 9-43.

Marin, D.; Castro, M.; Santana, P. (Coord.). (2018). Research and Innovation in Digital Education. @tic revista d'educació educativa (Monográfico), 20.

Martinez, N.; Valls, R.; Piñeda, F. (2009). El uso del libro de texto de Historia de España en Bachillerato: diez años de estudio, 1993-2003, y dos reformas (LGE-LOGSE). Didáctica de las Ciencias Experimentales y Sociales, 23, p. 3-35.

Munakata, Kazumi. (2012). Livro didático: alguns temas de pesquisa. Rev. Bras. Hist. Educ., Campinas-SP, v. 12, n. 3 (30), p. 179-197, set./dez.

Rodríguez Rodríguez, Jesús; Area Moreira, Manuel. (2017). (Coords). De los libros de texto a los materiales didácticos digitales. (Monográfico). Relatec, 16 (2).

Rodríguez Rodríguez, J.; Martínez Bonafé, J.; Braga Garcia, T. (2016). (Coords.). Entre libros de texto impresos y libros de texto digitales: debate y desafío. Profesorado. Revista de Currículum y Formación del Profesorado. (Monográfico), 20, 1.

Rodríguez Rodríguez, J.; Bruillard, E.; Horsley, M. (Eds.). (2015). Digital Textbooks: What's New. Santiago de Compostela: IARTEM/Universidade de Santiago de Compostela.

Rodríguez Rodríguez, J.; Garcia, T. B.; Bruillard, E. (Eds.). (2019). IARTEM 1991-2016: 25 years developing textbook and educational media research. Kongsberg: IARTEM. 